\title{
Impact of human CA8 on thermal antinociception in relation to morphine equivalence in mice
}

\author{
Eugene S. Fu ${ }^{a}$, Diana M. Erasso ${ }^{a}$, Gerald Z. Zhuang ${ }^{a}$, Udita Upadhyay, \\ Mehtap Ozdemir ${ }^{\mathrm{a}}$, Timothy Wiltshire ${ }^{\mathrm{d}}$, Konstantinos D. Sarantopoulos ${ }^{\mathrm{a}}$, \\ Shad B. Smith ${ }^{\mathrm{e}}$, William Maixner ${ }^{\mathrm{e}}$, Eden R. Martin ${ }^{\mathrm{b}, \mathrm{c}}$ and Roy C. Levitt ${ }^{\mathrm{a}, \mathrm{b}, \mathrm{c}}$
}

\begin{abstract}
Recently, we showed that murine dorsal root ganglion (DRG) Car8 expression is a cis-regulated eQTL that determines analgesic responses. In this report, we show that transduction through sciatic nerve injection of DRG with human wild-type carbonic anhydrase-8 using adeno-associated virus viral particles (AAV8-V5-CA8WT) produces analgesia in naive male $C 57 \mathrm{BL} / 6 \mathrm{~J}$ mice and antihyperalgesia after carrageenan treatment. A peak mean increase of about $4 \mathrm{~s}$ in thermal hindpaw withdrawal latency equaled increases in thermal withdrawal latency produced by $10 \mathrm{mg} / \mathrm{kg}$ intraperitoneal morphine in these mice. Allometric conversion of this intraperitoneal morphine dose in mice equals an oral morphine dose of about $146 \mathrm{mg}$ in a 60-kg adult. Our work quantifies for the first time analgesia and antihyperalgesia in an inflammatory pain model after DRG transduction by CA8 gene
\end{abstract}

therapy. NeuroReport 28:1215-1220 Copyright (c) 2017 The Author(s). Published by Wolters Kluwer Health, Inc.

NeuroReport 2017, 28:1215-1220

Keywords: analgesia, antihyperalgesia, antinociception, CA8 gene therapy, carbonic anhydrase-8, inflammatory pain, morphine

aDepartment of Anesthesiology, Perioperative Medicine and Pain Management, bJohn P. Hussman Institute for Human Genomics, 'John T. Macdonald Foundation, Department of Human Genetics, University of Miami Miller School of Medicine, Miami, Florida, ${ }^{\mathrm{d}}$ Center for Pharmacogenetics and Individualized Therapy, University of North Caroline, Eshelman School of Pharmacy, Chapel Hill and ${ }^{e}$ Department of Anesthesiology, Duke University School of Medicine, Durham, North Carolina, USA

Correspondence to Roy C. Levitt, MD, Room 8052A (R-371), Rosenstiel Medical Sciences Building, Department of Anesthesiology, Perioperative Medicine and Pain Management, University of Miami Miller School of Medicine, Miami, FL 33136, USA

Tel: + 1305 2431278; fax: + 1305243 1373; e-mail: rlevitt@med.miami.edu

Received 16 July 2017 accepted 9 August 2017

the expression vector encoding Car8 wild-type (WT) cDNA to produce antinociception. However, this finding is 'paradoxical' with respect to the mechanism of analgesia seen with morphine. The Car8 protein is known to inhibit inositol 1,4,5-triphosphate receptor-1 (ITPR1) to decrease calcium release [6], whereas morphine depends on increased ITPR1-dependent calcium release to produce analgesia [7]. In another study, we addressed the morphine 'paradox' in which we found that murine DRG Car8 gene expression is highly variable across inbred strains of mice and genetically regulates the analgesic effects of morphine in an antagonistic manner [8]. We surmised that the antagonism between Car8 expression and morphine may be related to their opposing effects on ITPR1-mediated calcium release [8].

In this report, we extended our previous findings [6] to evaluate the potential of the human form of carbonic anhydrase-8 $(C A 8)$ to produce thermal antinociception in naive $\mathrm{C57BL} / 6 \mathrm{~J}$ male mice. To quantify any resultant analgesia, we performed $\mathrm{SN}$ injections of AAV8 containing a vector encoding V5-CA8WT compared with injections containing vector encoding V5-CA8MT with the S100P null MT [9]. We report herein that AAV8-V5-CA8WT significantly increased thermal baseline withdrawal latencies before carrageenan inflammation, indicating analgesia. AAV8-V5-CA8WT
This is an open-access article distributed under the terms of the Creative Commons Attribution-Non Commercial-No Derivatives License 4.0 (CCBY-NCND), where it is permissible to download and share the work provided it is properly cited. The work cannot be changed in any way or used commercially without permission from the journal. 
also attenuated decreases in withdrawal latencies after carrageenan inflammation, indicating antihyperalgesia. We further showed quantification of these analgesic and antihyperalgesic responses in morphine equivalents.

\section{Materials and methods Animals}

All experiments and procedures were carried out in accordance with the Guide for the Care and Use of Laboratory Animals and the current guidelines for investigation of experimental pain in conscious animals [10,11]. This protocol was approved by the Animal Care and Use Committee of the University of Miami. Male adult C57BL/ $6 \mathrm{~J}$ mice, 12-14 weeks of age and weighing 30-35 g, were obtained from Jackson Laboratories (Bar Harbor, Maine, USA). Mice were kept in a home cage environment with access to food and water ad libitum. Animals were allowed to acclimatize for at least 7 days and were housed in a $12-12 \mathrm{~h}$ light-dark cycle in a virus/antigen-free facility with controlled humidity and temperature.

\section{Generation and SN injection of AAV8-V5-CA8 viral particles}

Generation and SN injection of AAV8 viral particles is described in our previous work [6]. These viral constructs express human $C A 8 W T$ or $M T C A 8$ vectors with V5 tag. The S100P mutation in the MT vector is associated with proteasome-mediated degradation that represents a null mutation comparable to the causal Car8 deletion mutation of the waddles mouse [6,9]. After SN exposure, $1.5 \mu$ l of about 2.0E14 genome copies/ml viral particles containing V5-CASWT and V5-CA8MT were injected into the SN. The negative control group received saline injections through $\mathrm{SN}$ under anesthesia.

\section{Mouse model of inflammatory pain in relation to neurobehavioral testing}

A volume of $15 \mu \mathrm{l}$ of $1 \% \lambda$ carrageenan (Sigma; SigmaAldrich Corp., St. Louis, Missouri, USA) in saline was subcutaneously injected into the plantar surface of left hindpaw. Thermal sensitivity was measured using heat according to the Hargreaves' test, to obtain threshold calculations, respectively. Tests were performed in a quiet room with daylight-like illumination. Animals were habituated to the behavioral room and apparatus for at least $60 \mathrm{~min}$ for 1 week before a blinded investigator collected baseline data. The thermal sensitivity test was performed using an IITC Plantar Antinociception Meter apparatus (IITC Life Sciences, Los Angeles, California, USA) with a plastic box placed on a glass plate of constant temperature $\left(30^{\circ} \mathrm{C}\right)$. The mouse plantar surface was exposed to a beam of radiant heat to induce paw withdrawal. The intensity of the Hargreaves' device was adjusted to obtain average values of $5-9 \mathrm{~s}$ in control mice, with a maximum of $20 \mathrm{~s}$ as cutoff to prevent potential injury. The latency time (in seconds) from the onset of the intense light beam to paw withdrawal was defined as the withdrawal latency of the paw. Two consecutive tests were averaged to establish the paw withdrawal latency.

\section{Morphine dosing regimen in relation to neurobehavioral testing}

For the dose-response assessment, we chose to study doses of saline (vehicle), 1, 3, 10, 30, and $60 \mathrm{mg} / \mathrm{kg}$. The thermal responses of male naive $\mathrm{C} 57 \mathrm{BL} / 6 \mathrm{~J}$ mice were measured at 30-60 min after a single dose of intraperitoneal saline or morphine administration. This time frame was chosen based on the morphine response findings from Dogrul and Seyrak [12]. On a further note, the $30-60 \mathrm{~min}$ time frame was required to measure thermal responses after saline or morphine administration, and reflects the acclimation time needed in the behavioral device. Moreover, we show that the maximal response to morphine resides within this window. These morphine doses were selected based on the findings by Wilson et al. [13] in which they found that maximal thermal antinociception occurred at doses less than $100 \mathrm{mg} / \mathrm{kg}$ in C57BL/6J mice using a tail immersion assay.

Thermal sensitivity was measured in these mice using the Hargreaves' assay, as previously described. The thermal latency data in the morphine dose-response assessment are presented after normalization to vehicle (saline). Normalization of the data entailed subtraction of the saline response to adjust for environmental effects due to intraperitoneal injections in nonanesthetized mice.

\section{Statistical analysis}

The sample size was $n=8$ per group for all experiments and the total number of mice studied was $N=72$. For data presented in Fig. 1, a Tukey's multiple-comparison posthoc test of a repeated two-way analysis of variance (treatment $\times$ time) was used to analyze these data (IBM SPSS Statistics 24, Armonk, New York, USA). Time was used as the repeated measure factor to determine main effects of treatment, time, and the interaction. Tests of betweensubject effects were performed to show an observed power of 1.000 (computed using $\alpha=0.05$ ), which include assumption of sphericity, Greenhouse-Geisser correction, and Huynh-Feldt correction. IBM SPSS Statistics 24 was modified to directly calculate the significance between groups at each time point incorporating a Bonferroni's correction.

Power was estimated using a two-side two-sample $t$-test using GraphPad StatMate 2.00 software (La Jolla, California, USA) to determine sample size for data presented in Fig. 2. These calculations were in agreement with other studies $[14,15]$. This power analysis estimates that a group sample size of $n=8$ is expected to achieve $95 \%$ power with a significant level $(\alpha)$ of 0.05 . The morphine dosing data are expressed as mean \pm SEM. All data were normalized to saline response. Normalized data 


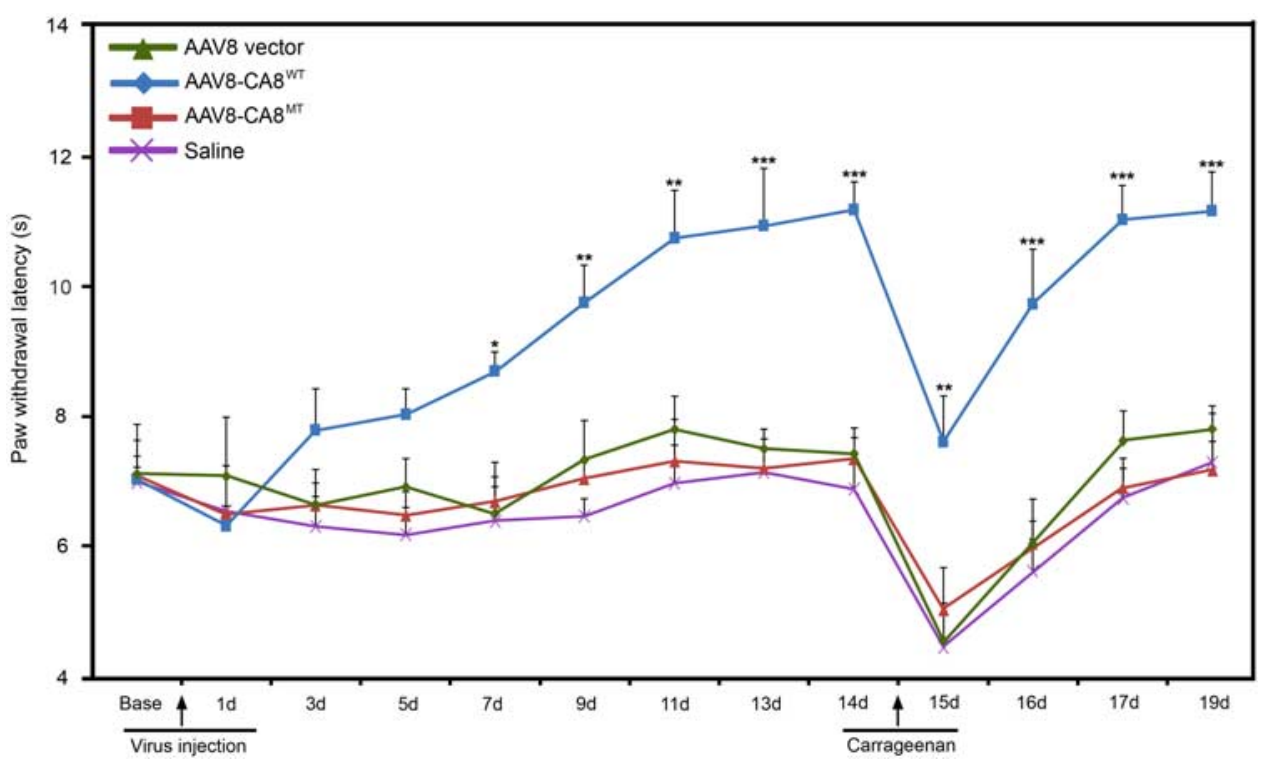

Gene transfer of AAV8-V5-CA8WT vector through sciatic nerve (SN) injection in a carrageenan inflammatory pain model produces analgesia and antihyperalgesia. Thermal latencies were measured at baseline and after intrasciatic saline or viral particle injection. Mice receiving SN injections of AAV8-V5-CA8WT (wild type) vector (1.5 $\mu \mathrm{l}, 2.65 \mathrm{E} 14$ genome copies $/ \mathrm{ml}$ ) had increased basal thermal latencies after day 7 compared with saline-treated mice. After carrageenan injections on day 14, mice in the AAV vector and AAV8-V5-CA8MT (mutant) vector groups had reductions in latency values markedly below baseline on day 15 and day 16 , indicating failure to protect from carrageenan-related hyperalgesia. In contrast, after carrageenan injections on day 14, AAV8-V5-CA8WT-treated mice showed an attenuated reduction in thermal latencies on day 15 and day 16 , in which these latency values did not differ from baseline values. AAV8-V5-CA8WT therapy restored thermal latencies to above baseline on day 17 and day 19 , indicating a protective effect in response to inflammatory hyperalgesia $\left(N=8 ;{ }^{*} P<0.05,{ }^{* \star} P<0.01,{ }^{* \star \star} P<0.001\right.$, when compared with saline-treated mice).

Fig. 2

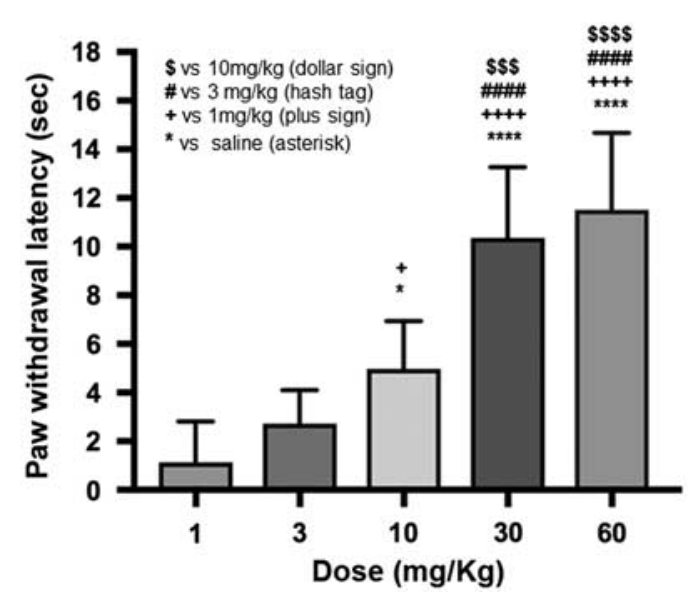

Morphine dose-response in naive mice for thermal withdrawal latencies. Eight 10-week-old naive male C57BL/6J mice were injected intraperitoneally in each group with morphine at each dose diluted in saline. Saline was used as vehicle control and all results were normalized to saline response. Each bar denotes mean \pm SEM group response $\left(N=8 ;{ }^{\star \star \star \star \star} P<0.0001,{ }^{\star} P<0.05\right.$ vs. saline controls, ${ }^{++++} P<0.0001$, ${ }^{+} P<0.05$ vs. $1 \mathrm{mg} / \mathrm{kg}$ group; \#\#\#\# $P<0.0001$ vs. $3 \mathrm{mg} / \mathrm{kg}$ group; $\$ \$ \$ P<0.0001, \$ \$ \$ P<0.001$ vs. $10 \mathrm{mg} / \mathrm{kg}$ group).

were analyzed for statistical significance by one-way analysis of variance followed by Tukey's multiplecomparison post-hoc test for multiple comparisons (three or more groups) with one variance.

\section{Results \\ DRG CA8 overexpression alters thermal nociception before and after carrageenan inflammation}

We constructed vectors containing the WT CA 8 cDNA with a V5 tag (V5-CA8WT) and CA8 MT cDNA sequence (S100P), which served as a negative control (V5-CA8MT) $[8,9]$. We assessed the effects of V5-CA8 overexpression on thermal nociceptive thresholds for 19 days after $\mathrm{SN}$ injection and DRG transduction before and after carrageenan injection and inflammation in the hindpaw of naive C57BL/6J mice (Fig. 1).

Before saline or viral $\mathrm{SN}$ injections, baseline thermal latency in naive mice was $\sim 7 \mathrm{~s}$ (mean \pm SD: $7.34 \pm 1.09 \mathrm{~s}$ ). As shown in Fig. 1, thermal withdrawal latencies were measured in saline-treated and vector-treated mice. Differences in thermal withdrawal latencies were found between groups $[F(3,28)=21.48, P<0.001]$ and across the 13 time points $[F(12,336)=20.04, P<0.001]$. There were also significant interactions between time and group $[F(36,336)=4.37, P<0.001]$. Bonferroni's pairwise comparisons of time $\times$ group interaction indicated that there were no differences between groups at baseline, day 1 , day 3 , and day 5. Withdrawal latencies in the AAV8-V5-CA8WT group were greater than the saline group at all time points after day 7 and after carrageenan application. Withdrawal latencies in the AAV8 empty vector and AAV8-V5-CA8MT 
groups did not differ from saline treatment at all time points before and after carrageenan treatment.

On day $14, A A V 8-V 5-C A 8 W T$ treatment increased Hargreaves' thermal latency values by about $4 \mathrm{~s}$ compared with baseline values in the naive state (about $11 \mathrm{~s}$ compared with $7 \mathrm{~s}$ ). Thermal latencies did not increase in saline, empty vector, or AAV8-V5-CA8MT-treated groups. Following carrageenan inflammation, the transduction of the mouse DRG with V5-CA8WT prevented a decrease in thermal thresholds below baseline values, with restoration of elevated thermal latencies of about $4 \mathrm{~s}$ above baseline by day 19. Conversely, $\mathrm{SN}$ administration of saline, virus containing empty vector, and AAV8-V5-CABMT provided no protection from a decrease in thermal thresholds from baseline following carrageenan inflammation.

\section{Effect of morphine on thermal nociception before carrageenan inflammation}

The raw baseline thermal latency for naive mice in Fig. 2 was $\sim 7 \mathrm{~s}($ mean \pm SD: $6.87 \pm 0.65)$, which were similar to the unadjusted baseline thermal latency for naive mice studied. Figure 2 shows normalized thermal latencies for intraperitoneal vehicle responses (saline) by subtracting the saline component. After normalization, we found significant increases in thermal withdrawal latencies from mice receiving 10,30 , and $60 \mathrm{mg} / \mathrm{kg}$ of morphine compared with saline-treated mice. Mice receiving the highest dose of morphine $(60 \mathrm{mg} / \mathrm{kg})$ had increases in thermal withdrawal latencies compared with mice receiving 1,3 , and $10 \mathrm{mg} / \mathrm{kg}$ of morphine, but not compared with mice receiving $30 \mathrm{mg} / \mathrm{kg}$. On the basis of this analysis, we were able to assess the amount of parenteral morphine required to increase thermal withdrawal latencies in these naive mice and before carrageenan inflammation. In addition to normalizing the data, we also calculated maximal percentage effectiveness (\%MPE) using the formula: (measured thermal latency-saline baseline latency)/[cutoff latency (20 s) - baseline latency] [13]. We found a progressive increase in \%MPE in relation to the dose, in which the \%MPE was $7.48 \%$ for the $1 \mathrm{mg} / \mathrm{kg}$ group, $20.17 \%$ for the $3 \mathrm{mg} / \mathrm{kg}$ group, $36.28 \%$ for the $10 \mathrm{mg} / \mathrm{kg}$ group, $78.86 \%$ for the $30 \mathrm{mg} / \mathrm{kg}$ group, and $86.20 \%$ for the $60 \mathrm{mg} / \mathrm{kg}$ group.

\section{Scaling of morphine from mice to humans based on the dose response of morphine on thermal nociception}

The amount of morphine administered in mice to produce antinociception (increase in thermal response above baseline) is known as the mouse equivalent dose (Table 1). By obtaining mouse morphine equivalents, these data can be translated to a human equivalent dose by allometric conversion that depends on a formula that accounts for body surface area coefficients in humans and mice [16]. As shown in Table 1, we can extrapolate the mouse equivalent dose for thermal somatosensory responses to clinical oral morphine dosing in humans.
Using this approach, we estimated that increases in thermal withdrawal latencies on the order of $4 \mathrm{~s}$, as seen with AAV8-V5-CASWT therapy, corresponds to a dose equivalent to about $10 \mathrm{mg} / \mathrm{kg}$ of intraperitoneal morphine (Fig. 2). We found that $10 \mathrm{mg} / \mathrm{kg}$ of systemic morphine in mice translates to human equivalent dose of $0.81 \mathrm{mg} / \mathrm{kg}$, $48.65 \mathrm{mg}$ of parental morphine, and $145.95 \mathrm{mg}$ of oral morphine in a $60-\mathrm{kg}$ adult (Table 1).

\section{Discussion}

In a previous study, we noted that thermal antinociception in the noninflammatory state after Car8 gene therapy persisted for at least 28 days [6]. In this report, we show that $\mathrm{SN}$ administration in naive inbred mice and DRG transduction by using AAV8-V5-CA8WT gene therapy produces profound analgesia as demonstrated by a maximal increase of thermal latencies about $4 \mathrm{~s}$ above baseline (Fig. 1). In addition, we show that CA8 therapy produced antihyperalgesia by preventing a drop in thermal latencies below baseline in response to carrageenan inflammation along with restored thermal baselines to $4 \mathrm{~s}$ above baseline by day 19 . These findings support the concept that $C A 8$ gene therapy provides prolonged analgesia before or after the onset of inflammatory pain.

As aforementioned, both CA8 and morphine have 'paradoxical' effects on intracellular calcium release, even though both can produce antinociception. Morphine antinociception has been attributed to increased calcium release through ITPR1 binding of inositol 1,4,5-triphosphate (IP3) [7]. In contrast, we demonstrated that murine AAV8-V5-Car8WT showed inhibition of ITPR1-mediated cytosolic calcium release in conjunction with decreased thermal hypersensitivity [6]. We also found that greater DRG Car8 expression antagonizes morphine analgesia concomitant with regulation of morphine-induced ITPR-mediated calcium release [8]. The disadvantage of $\mu$ opioids, such as of morphine, is the development of analgesic tolerance seen after morphine administration [14], which may involve pathways that increase intracellular calcium release such as IP3 binding to IP3 receptors [17]. Further studies are warranted to determine whether CA8 therapy, which involves inhibition of ITPR1-mediated calcium release, could be advantageous in providing antinociception without the development of analgesic tolerance.

We sought to determine the amount of morphine equivalents needed to produce thermal antinociception and correlate that with thermal antinociception associated with $C A 8$ therapy. The degree of thermal antinociception in mice in response to morphine was assessed by dose-response analysis (Fig. 2). On the basis of allometric modeling by Reagan-Shaw et al. [16] among various species, the proper translation of animal dosing to human dosing entails the use of body surface area in addition to weight. Using the morphine dose-response data collected herein in naive male C57BL/6J mice, we show by 
Table 1 The effect of increasing parental doses of morphine on mouse hindpaw thermal withdrawal latency, with allometric conversion to human oral morphine dosing

\begin{tabular}{|c|c|c|c|c|c|}
\hline Increase in mouse hindpaw thermal withdrawal latency (s) & 0.99 & 2.63 & 4.71 & 10.21 & 11.16 \\
\hline Mouse equivalent dose of morphine $(\mathrm{mg} / \mathrm{kg})$ & 1 & 3 & 10 & 30 & 60 \\
\hline Human equivalent dose of morphine $(\mathrm{mg} / \mathrm{kg})$ & 0.08 & 0.24 & 0.81 & 2.43 & 4.86 \\
\hline Parental morphine dose in a $60-\mathrm{kg}$ adult $(\mathrm{mg})$ & 4.86 & 14.59 & 48.65 & 145.95 & 291.89 \\
\hline Oral morphine dose in a $60 \mathrm{~kg}$ adult $(\mathrm{mg})$ & 14.59 & 43.78 & 145.95 & 437.84 & 875.68 \\
\hline
\end{tabular}

Mouse equivalent dose (MED) of morphine is obtained by determining the dose of morphine required to produce an analgesic effect as manifested by increases in mechanical or thermal thresholds above baseline. Human equivalent dose (HED) of morphine is obtained by dose translation from mouse to humans using $K_{\mathrm{m}}$ factor coefficients. On the basis of allometric modeling by Reagan-Shaw et al. [16], the $K_{\mathrm{m}}$ factor is derived by dividing the average body weight in kg of an animal species to its body surface area in $\mathrm{m}^{2}$, in which $K_{\mathrm{m}}$ mouse $=3$ and $K_{\mathrm{m}}$ human $=37$. HED $(\mathrm{mg} / \mathrm{kg})=\mathrm{MED}(\mathrm{mg} / \mathrm{kg}) \times\left(\mathrm{mouse} K_{\mathrm{m}} / \mathrm{human} K_{\mathrm{m}}\right)$. Parental morphine dose in a $60-\mathrm{kg}$ adult human is calculated after obtaining the HED. Oral morphine dose is approximately three times the parental morphine dose in a 60-kg adult.

allometric conversion that $C A 8$ analgesia from this experiment provided increases in thermal latency on the order of $4 \mathrm{~s}$, which translates to morphine dosing equivalents of about $146 \mathrm{mg}$ of oral morphine in a $60-\mathrm{kg}$ adult human. These adult morphine quantities would exceed the recommended dosing maximum of $50-120 \mathrm{mg}$ of daily oral morphine equivalents based on Centers for Disease Control [18] and Washington State Interagency Guidelines [19] on opioid dosing in chronic noncancer pain patients.

By establishing the relationship between morphine dosing and the degree of analgesia and antihyperalgesia associated with $C A 8$ gene therapy, we extrapolated the potential clinical significance of $C A 8$ gene therapy to morphine equivalents. However, there are several limitations with this strategy. We assessed morphine equivalents in a single inbred strain of male mice before inflammatory challenge. Nonetheless, the increases in thermal latencies observed after AAV8-V5-CA8WT therapy clearly persist after carrageenan inflammation, as compared with saline, empty vector, and MT. Thus, it appears that measuring morphine analgesic equivalents before the onset of carrageenan inflammation should still be relevant. In addition, inbred strains of mice differ in analgesic response by sex and strain, limiting the extrapolation of specific findings [20]. For example, C57BL/6 mice only achieve about $75 \%$ maximal percentage of thermal antinociception with morphine compared with BALB/cByJ mice, which had the highest degree of maximal percentage antinociception on the order of $90-100 \%$ [13]. Therefore, this strain appears relevant to test our general hypothesis.

\section{Conclusion}

We determined that AAV8-V5-CASWT SN injection produces profound analgesia in mice before and after severe inflammatory pain, as assessed in morphine equivalents. Further assessment of the safety profile of AAV8-V5-CA8WT therapy in mice will entail various assays to rule out tissue toxicity and neurobehavioral abnormalities. Future research is also needed in understanding the role of known analgesics such as morphine and clonidine in relation to CA8 analgesia, inflammation, and calcium release.

\section{Acknowledgements}

The authors thank Dr D. A. Lubarsky for his generous input and support.

This work was funded by grants from the NIDCR R01DE022903; the NIDA R031410; and funding from the Department of Anesthesiology, Perioperative Medicine, and Pain Management, University of Miami Miller School of Medicine, Miami, Florida, USA.

\section{Conflicts of interest}

There are no conflicts of interest.

\section{References}

1 Institute of Medicine of the National Academies, Committee on Advancing Pain Research, Care, and Education. Relieving pain in America: a blueprint for transforming prevention, care, education, and research. Washington, DC: National Academies Press (US); 2011. p. 1.

2 Volkow ND, McLellan T. Opioid abuse in chronic pain - misconceptions and mitigation strategies. N Engl J Med 2016; 374:1253-1263.

3 Rudd RA, Zibbell JE, Gladden RM. Increases in drug and opioid overdose deaths - United States, 2000-2014. Morb Mortal Wkly Rep 2016; 64:1378-1382.

4 Skolnick P, Volkow ND. Re-energizing the development of pain therapeutics in light of the opioid epidemic. Neuron 2016; 92:294-297.

5 Miller G. Is pharma running out of brainy ideas? Science 2010; 329:502-504.

6 Zhuang GZ, Keeler B, Grant J, Bianchi L, Fu ES, Zhang YP, et al. Carbonic anhydrase-8 regulates inflammatory pain by inhibiting the ITPR1-cytosolic free calcium pathway. PLoS One 2015; 10:e0118273.

7 Aoki T, Narita M, Ohnishi O, Mizuo K, Yajima Y, Suzuki T. Disruption of the type 1 insoitol 1,45-triphosphsate receptor gene suppresses the morphineinduce antinociception in the mouse. Neurosci Lett 2003; 350:69-72.

8 Levitt RC, Zhuang GY, Kang Y, Erasso DM, Upadhyay U, Ozdemir M, et al. Car8 dorsal root ganglion expression and genetic regulation of analgesic responses are associated with a cis-eQTL in mice. Mamm Genome 2017; doi: 10.1007/s00335-017-9694-7.

9 Turkmen S, Guo G, Garshasbi M, Hoffmann K, Alshalah AJ, Mischung C, et al. CA8 mutations cause a novel sykndrome characterized by ataxia and mild mental retardation with predisposition to quadrupedal gait. PLoS Genet 2009; 5:e1000487.

10 National Academies Press. Guide for the care and use of laboratory animals, 8th ed. Washington, DC: National Academies Press (US); 2011.

11 Zimmermann M. Ethical guidelines for investigations of experimental pain in conscious animals. Pain 1983; 16:109-110.

12 Dogrul A, Seyrak M. Systemic morphine produce antinociception mediated by spinal 5-HT7, but not 5-HT1A and 5-HT2 receptors in the spinal cord. $\mathrm{Br} J$ Pharmacol 2006; 149:498-505.

13 Wilson SG, Smith SB, Chesler EJ, Melton KA, Haas JJ, Mitton B, et al. The heritability of antinociception: common pharmacogenetic mediation of five neurochemically distinct analgesics. J Pharmacol Exp Ther 2003; 304:547-559.

14 Elhabazi K, Ayachi S, llien B, Simonin F. Assessment of morphine-induced hyperalgesia and analgesic tolerance in mice using thermal and mechanical nociceptive modalities. J Vis Exp 2014; 89:e51264. 
15 Elmer GI, Pieper JO, Negus SS, Woods JH. Genetic variance in nociception and its relationship to the potency of morphine-induced analgesia in thermal and chemical tests. Pain 1998; 75:129-140.

16 Reagan-Shaw S, Nihal M, Ahmad N. Dose translation from animal to human studies revisited. FASEB J 2008; 22:659-661.

17 Smith FL, Lohmann AB, Dewey DL. Involvement of phospholipid signal transduction pathways in morphine tolerance in mice. Br J Pharmacol 1999; 128:220-226.
18 Dowell D, Haegerich TM, Chou R. CDC guideline for prescribing opioids for chronic pain - United States. JAMA 2016; 315:1624-1645.

19 Skaer TL, Nwude AC. Opioid prescribing laws and emergency department guidelines for chronic non-cancer pain in Washington State. Pain Pract 2016; 16:642-647.

20 Lariviere WR, Wilson SG, Laughlin TM, Kokayeff A, West EE, Adhikari SM et al. Heritability of nociception. III. Genetic relationships among commonly used assays of nociception and hypersensitivity. Pain 2002; 97:75-86. 